\title{
On the Value of an NCA Journal Dedicated to Communication and Technology: Quantifying a Missed Opportunity
}

\author{
Stephen A. Rains
}

The University of Arizona

The introduction of virtually any new journal sponsored by the National Communication Association (NCA) is likely to prompt questions among scholars in our field. Why does NCA need yet another journal? At the end of 2019, NCA already sponsored 11 journals covering a range of topics from communication education and first amendment studies to intercultural and applied communication. What possible justification could exist to dedicate a twelfth journal to communication and technology? Although there are a number of ways such questions might be answered, one worthwhile approach is to consider contemporary publication practices to determine whether a new journal is warranted. To this end, I report an analysis of publication patterns in NCA and International Communication Association (ICA) journals from 2000-2015. Using keywords in article abstracts as a means to identify technology articles, I track the prevalence of communication and technology articles over time and across journal types. The growth in communication and technology research in our field and differences in the prevalence of technology articles appearing in NCA and ICA journals are then used to highlight the substantial need for an NCA journal dedicated to this topic. As will be shown, the absence of such a journal represents a considerable missed opportunity for NCA to embrace cutting-edge research trends in our discipline and become a leading voice in communication and technology scholarship.

\section{Identifying the Prevalence of Communication and Technology Research}

In order to track trends in communication and technology research, the Communication and Mass Media database was used to extract citation information and abstracts for every article published in the nine journals sponsored by $\mathrm{NCA}^{1}$ that focus on original scholarship and the six journals sponsored by $\mathrm{ICA}^{2}$ from 2000 $2015(N=5,187)$. In order to isolate original research, all entries consisting of three or fewer pages as well as entries explicitly labeled as book reviews were excluded. This step was necessary to omit entries like announcements, errata, and other miscellany that appear in journals and are not original scholarship. The resulting sample of 4,913 abstracts were then evaluated to identify articles addressing communication and technology. 
Communication and technology articles were operationally defined as articles with abstracts that contained one or more of 20 keywords: technolog*, online, online, internet, computer, CMC, social media, social networking, blog*, electronic, mail, instant messaging, phone, digital, web, mobile, medium, channel, virtual, cyber*. These terms were selected because they reflect commonly-studied technologies or central constructs in communication and technology research. As such, articles that used these terms were likely to address topics in the domain of communication and technology. A total of 1,336 abstracts included one or more of the 20 keywords and were considered communication and technology articles in the following analyses.

In order to evaluate the prevalence of communication and technology research, the raw numbers of technology articles published each year in ICA and NCA journals were plotted. As illustrated in Figure 1, publication rates for communication and technology articles increased steadily in both NCA and ICA journals. During 2000, NCA and ICA journals each published 13 technology articles. Publication rates increased to 53 articles for NCA and 76 articles for ICA during 2015. Despite the general increase, there was a noticeable difference between the two types of journals. From 2000 until 2015, ICA published an average of 54.1 $(S D=29.7)$ technology articles per year compared to $29.4(S D=12.6)$ articles per year published by NCA. This yearly difference translates into a total of almost 400 more technology articles published in ICA journals than NCA journals during the sampling frame.

In addition to the raw number of articles published, the prevalence of communication and technology articles as a proportion of all articles published in a set of journals was evaluated. As illustrated in Figure 2, both NCA and ICA journals have dedicated increasing journal space to communication and technology articles. During 2015, communication and technology articles occupied almost twice

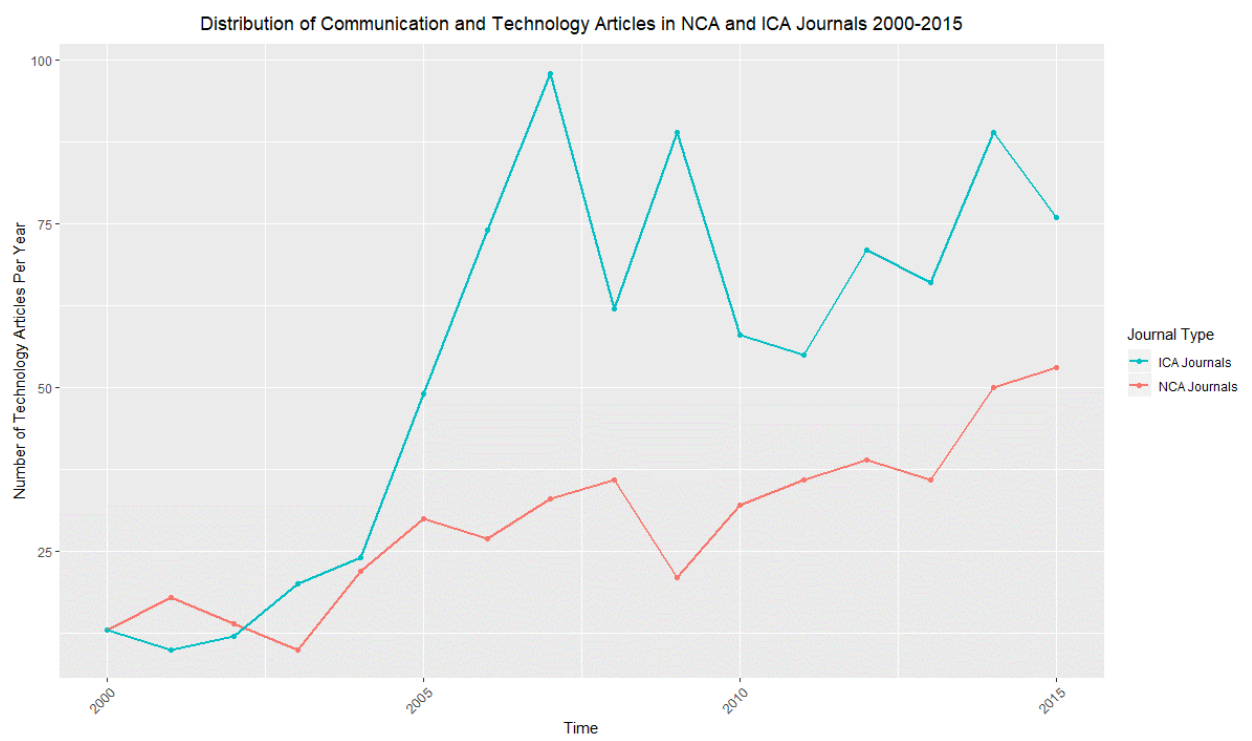

Figure 1. Communication and technology article publication rates in NCA and ICA journals. 


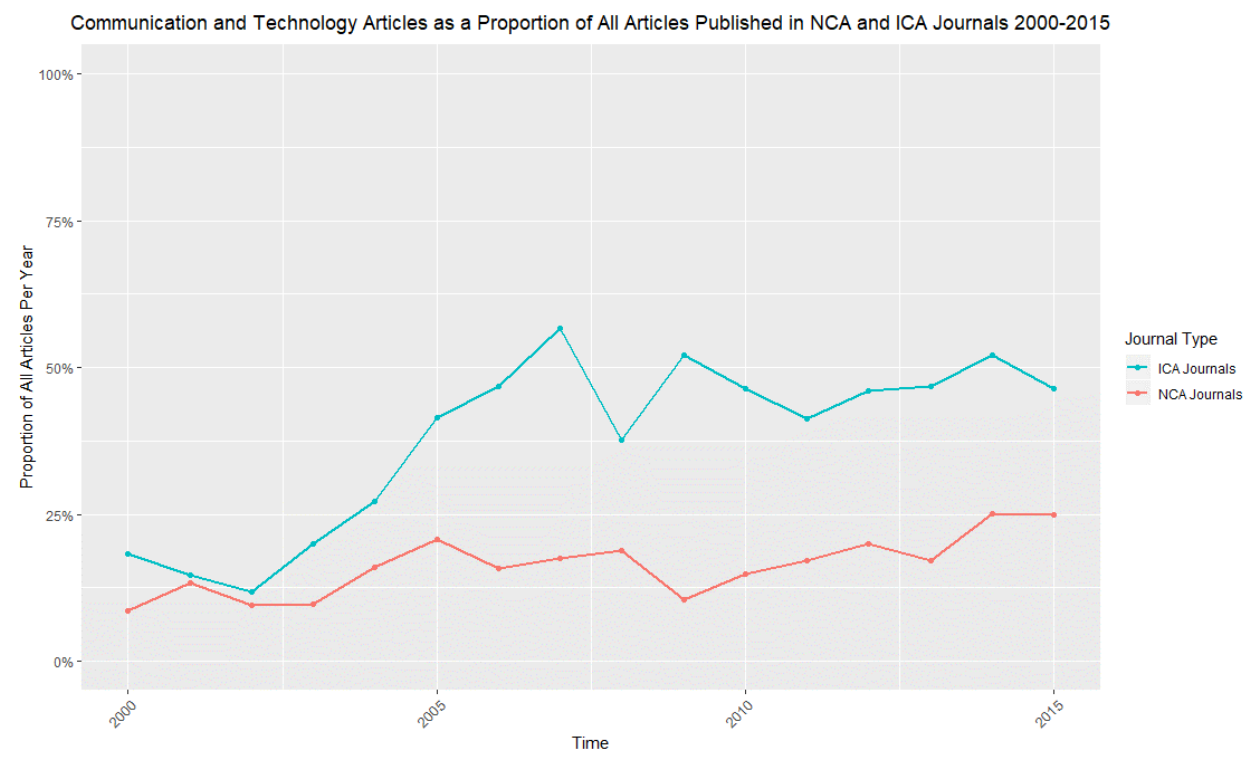

Figure 2. Technology articles relative to all articles published in NCA and ICA journals.

the space at NCA and ICA journals as they did during 2000. A significant gap remained, however, in the proportion of space dedicated between the two sets of journals. Whereas an average of $37.9 \%(S D=14.6 \%)$ of articles published each year in ICA journals addressed communication and technology, NCA journals dedicated an average of $16.2 \%(S D=5.1 \%)$ of yearly articles to this topic. Almost half of all articles published in ICA journals during 2015 addressed communication and technology.

\section{A Missed Opportunity and Redemption}

Several conclusions might be drawn from the preceding analyses about the need for and merits of a new journal dedicated to communication and technology. Perhaps the most obvious conclusion involves the significant growth in communication and technology scholarship. Between 2000 and 2015, 27.2\% of all articles appearing in journals sponsored by NCA and ICA had an abstract containing one of the 20 keywords related to communication and technology. To put this in perspective, one in every four articles appearing in NCA and ICA journals addressed communication and technology. To say that research on communication and technology has arrived would be an understatement. Communication and technology has become a significant and pervasive topic of study within our discipline.

A second conclusion is that communication and technology scholarship has not been evenly distributed between NCA and ICA journals. Although NCA and ICA journals published a similar number of communication and technology articles during the year 2000, the difference between journals sponsored by these two organizations grew considerably since that time. From 2000 to 2015, ICA published almost twice the number of communication and technology articles per 
year as NCA. That yearly difference accumulated to a total of 396 more articles published by ICA than NCA during the sampling frame.

It seems fair to question why ICA journals would publish such a markedly greater volume of technology research than NCA journals. Although there are important differences between ICA and NCA, these two organizations have numerous similarities. NCA and ICA both share a longstanding commitment to supporting high-quality scholarship about human communication. Both organizations sponsor well-regarded journals with relatively high impact factors. Finally, there is significant overlap in the subfields represented by each organization and membership. In short, there is no theoretical or substantive reason why ICA journals should necessarily publish substantially more communication and technology articles than NCA journals. Given their considerable similarities, communication and technology scholarship should be equally prevalent in journals sponsored by these two organizations.

There is, however, one structural difference between NCA and ICA that warrants consideration. By this point, I suspect that many readers may have isolated the source of the discrepancy in communication and technology articles between NCA and ICA journals. In the Journal of Computer-Mediated Communication (JCMC), ICA has a journal dedicated to publishing research examining the implications of communication technologies. Indeed, JCMC published 402 articles between 2000 and 2015 whose abstracts included one of the 20 communication and technology keywords examined in this project. The number of articles published by JCMC is almost identical to the 396 article cumulative difference between ICA and NCA journals from 2000 to 2015. The discrepancy in communication and technology articles published by NCA and ICA can be almost completely explained by JCMC.

Building on the two previous conclusions, a third and final conclusion that might be drawn from the analyses conducted for this essay involves the opportunity missed by NCA and a chance for redemption. Topics related to communication and technology are clearly among the most researched issues in our field since the year 2000. Despite this considerable interest, NCA journals have lagged well behind ICA in publishing communication and technology scholarship. Much of this discrepancy, however, can be traced to JCMC. Having a journal dedicated to communication and technology research allowed ICA to publish almost 400 more articles on this topic than NCA between 2000 and 2015. The absence of an NCA journal dedicated to the study of communication and technology is a missed opportunity. NCA has-until recently-missed the chance to become a flagbearer for communication and technology scholarship.

Rather than dwell on foregone opportunities, I believe that our time is better spent considering the future. The potential for redemption exists, and there is reason for optimism about communication and technology research at NCA moving forward. Human Communication \& Technology is the first NCA journal dedicated to the study of communication and technology. It represents a recognition and embrace of the dramatic growth in communication and technology scholarship since 2000. This new journal has the potential to level the playing field with ICA and put NCA at the forefront of communication and technology scholarship. The institutional credibility stemming from being sponsored by NCA will likely help attract the very best in communication and technology research. For individual 
scholars, it represents another reputable and visible outlet in which to publish one's ideas. Beyond the benefits to NCA and individual researchers, perhaps the most important contribution of this journal will make is to our collective work. In concert with JCMC, Human Communication \& Technology will add another dedicated forum for high-quality research that will ultimately serve to advance our collective understanding of communication and technology. There is cause for us to wholeheartedly welcome this new journal and be excited about what it will allow us to accomplish.

\section{Notes}

1. The nine NCA journals included: Communication and Critical/Cultural Studies, Communication Education, Communication Monographs, Critical Studies in Media Communication, First Amendment Studies, Journal of Applied Communication Research, Journal of International and Intercultural Communication, Quarterly Journal of Speech, and Text and Performance Quarterly.

2. The six ICA journals included: Annals of the International Communication Association, Communication, Culture, \& Critique, Communication Theory, Human Communication Research, Journal of Communication, and Journal of Computer-Mediated Communication. 